\title{
Hepatocellular carcinoma in non-alcoholic fatty liver disease- a review of an emerging challenge facing clinicians
}

\author{
Daniel Geh $^{1,2}$, Derek M. Manas ${ }^{2,3}$, Helen L. Reeves ${ }^{1,3}$ \\ ${ }^{1}$ Northern Institute for Cancer Research, ${ }^{2}$ Institute of Cellular Medicine, Newcastle University, Newcastle upon Tyne, UK; ${ }^{3}$ Hepatopancreatobiliary \\ Multidisciplinary Team, Newcastle upon Tyne Hospitals NHS Foundation Trust, Newcastle upon Tyne, UK \\ Contributions: (I) Conception and design: All authors; (II) Administrative support: None; (III) Provision of study materials or patients: None; (IV) \\ Collection and assembly of data: None; (V) Data analysis and interpretation: None; (VI) Manuscript writing: All authors; (VII) Final approval of \\ manuscript: All authors. \\ Correspondence to: Helen L Reeves. Professor of Liver Cancer, Northern Institute for Cancer Research, Framlington Place, Newcastle upon Tyne, \\ NE2 4HH, UK. Email: H.L.Reeves@newcastle.ac.uk.
}

Importance: Non-alcoholic fatty liver disease (NAFLD) is a rapidly growing cause of chronic liver disease and is becoming a leading cause of hepatocellular carcinoma (HCC) in many developed countries. This presents major challenges for the surveillance, diagnosis and treatment of HCC.

Objective: To discuss the clinical challenges faced by clinicians in managing the rising number of NAFLDHCC cases.

Evidence Review: MEDLINE, PubMed and Embase databases were searched using the keywords; NAFLD, HCC, surveillance, hepatectomy, liver transplantation, percutaneous ablation, transarterial chemoembolization (TACE), selective internal radiotherapy treatment (SIRT) and sorafenib. Relevant clinical studies were included.

Findings: Current HCC surveillance programmes are inadequate because they only screen for HCC in patients with cirrhosis, whereas in NAFLD a significant proportion of HCC develops in the absence of cirrhosis. Consequently NAFLD patients often present with a more advanced stage of HCC, with a poorer prognosis. NAFLD-HCC patients also tend to be older and to have more co-morbidities compared to HCC of other etiologies. This limits the use of curative treatments such as liver resection and orthotopic liver transplantation (OLT). Evidence suggests that although NAFLD-HCC patients who undergo liver resection or OLT have worse perioperative and short-term outcomes, overall long-term survival is comparable to HCC of other etiologies. This highlights the importance of careful patient selection, pre-habilitation and perioperative planning for NAFLD-HCC patients being considered for surgical treatment. Careful consideration is also important for non-surgical treatments, although the evidence supporting treatment selection is frequently lacking, as these patients tend to be poorly represented in clinical trials. Locoregional therapies such as percutaneous ablation and TACE may be less well tolerated and less effective in NAFLD patients with obesity or diabetes. The tyrosine kinase inhibitor sorafenib may also be less effective.

Conclusions and Relevance: This review highlights how international guidelines, for which NAFLD traditionally has made up a small part of the evidence base, may not be appropriate for all NAFLDHCC patients. Future guidelines need to reflect the changing landscape of HCC, by making specific recommendations for the management of NAFLD-HCC.

Keywords: Non- alcoholic fatty liver disease (NAFLD); hepatocellular carcinoma (HCC); liver transplantation; hepatectomy; catheter ablation; sorafenib; population surveillance

Submitted Jun 07, 2019. Accepted for publication Aug 21, 2019.

doi: $10.21037 / \mathrm{hbsn} .2019 .08 .08$

View this article at: http://dx.doi.org/10.21037/hbsn.2019.08.08 


\section{Introduction}

Hepatocellular carcinoma (HCC) is the most common primary liver cancer and usually occurs in the context of liver cirrhosis. Worldwide liver cancer is the fifth most common cancer and is ranked third in cancer related mortality (1). Whereas overall cancer mortality is generally decreasing, liver cancer is the most rapidly increasing cause of cancer mortality (2-5). In the United States between 2000 to 2016, mortality from liver cancer has increased by $43 \%(10.5$ to 15.0 per 100,000$)$ in men and by $40 \%$ (4.5 to 6.3 per 100,000$)$ in women $(6)$. Despite some improvement in surveillance and treatments, the overall 5 -year survival rate is around $15 \%$ (7). This growing burden of disease incidence and mortality has made HCC a significant public health concern.

The majority of HCC occurs in the context of liver cirrhosis. Globally chronic hepatitis B virus (HBV) and hepatitis $\mathrm{C}$ virus (HCV) related liver cirrhosis are the leading causes of HCC (8). In western countries, where viral hepatitis is less prevalent, alcohol-related liver disease (ARLD) and non-alcoholic fatty liver disease (NAFLD) are the leading causes of liver cirrhosis and HCC (9).

In the last few decades there has been an epidemic in obesity, dyslipidemia, hypertension and type 2 diabetes mellitus (T2DM). These entities, collectively known as the metabolic syndrome, increase the risk of atherosclerotic cardiovascular disease and NAFLD $(10,11)$. NAFLD has now become the most common cause of chronic liver disease with an estimated global prevalence of $25 \%$ (12). The rise of the metabolic syndrome, combined with the decline of viral hepatitis due to $\mathrm{HBV}$ vaccination and highly effective direct acting anti-virals to treat $\mathrm{HCV}$, has contributed to NAFLD becoming one of the leading causes of HCC in western countries (13-15). This brings major challenges with surveillance, diagnosis and management of HCC. We present the following article in accordance with the Narrative Review reporting checklist (available at https://hbsn.amegroups.com/ article/view/10.21037/hbsn.2019.08.08/rc).

\section{The increasing burden of HCC in NAFLD}

NAFLD is a spectrum of disease made up of hepatic steatosis, non-alcoholic steatohepatitis (NASH), fibrosis, cirrhosis and end stage liver disease. Around $25 \%$ of the adult population in the United States have evidence of NAFLD, with the majority having hepatic steatosis without evidence of inflammation or scarring. Of those with hepatic steatosis, reported studies suggest that around $25 \%$ will progress to NASH (6\% of the general population), of which a significant number will develop cirrhosis (16). NASH is characterized by steatosis, ballooning degeneration of hepatocytes, and inflammation. Progression to fibrosis occurs in $26-37 \%$ of those with biopsy proven NASH, of which around $9 \%$ reportedly develop cirrhosis over 5.6 years (17). NAFLD cirrhosis is currently estimated to have a prevalence of $1-2 \%$ in the United States and is emerging as the most common cause of cirrhosis worldwide (16). Of additional concern, the prevalence of NAFLD in the paediatric population is also increasing and is believed to be between $7.6 \%$ to $34.2 \%$ (18).

Not surprisingly, along with rise in NAFLD the prevalence of NAFLD associated HCC has also increased. The annual incidence of HCC is estimated to be $2.6 \%$ in patients with NAFLD cirrhosis (19). In total, NAFLD cirrhosis makes up around $10-14 \%$ of HCC cases in western countries and the incidence has increased by 9\% between 2004 to 2009 in the United States (20,21). The proportion of patients with NAFLD-HCC on liver transplant waiting lists increased from $2.1 \%$ to $16.2 \%$ in the United States between 2000 and 2016 (15). NAFLDHCC can also develop in the absence of cirrhosis, with the number of non-cirrhotic individuals with HCC increasing in prevalence and accounting for $25-45 \%$ of total NAFLDHCC cases $(13,22)$. Although HCC can develop in other etiologies of chronic liver disease in the absence of cirrhosis, NAFLD has a higher risk of non-cirrhotic HCC when compared to HCV and ALD (23). Due to the very large number of patients with NAFLD though, the absolute risk of developing NAFLD-HCC without cirrhosis is still small (24). The pathogenesis of NAFLD-HCC in this context is poorly understood, but chronic inflammation, hyperinsulinemia, adaptive immune responses, hepatic progenitor cell populations and genetic susceptibility may all play a role (25).

\section{Clinical features of NAFLD-HCC}

NAFLD-HCC tends to be diagnosed at a more advanced stage compared to HCC related to other etiologies $(22,26)$. A large Italian cohort demonstrated that NAFLD-HCC Barcelona Clinic Liver Cancer (BCLC) C tumours were significantly more common at the presentation of NAFLDHCC compared to HCV-HCC (21\% vs. 4\%, P<0.0001) (22). Both suboptimal HCC surveillance practice and NAFLDHCC arising in the absence of cirrhosis and hence no surveillance practice, are likely contributory factors $(22,26)$. 
Overall, patients with NAFLD-HCC have a worse prognosis compared to those with other etiologies, which most likely relates to the more advanced stage of disease at diagnosis, as well co-morbidities in patients with the metabolic syndrome, which limits the use of curative treatments. The Italian cohort study reported an average survival of 25.5 months in NAFLD-HCC compared to 33.7 months in HCV-HCC $(\mathrm{P}=0.017)$, but when adjusting for stage and curative treatments, this survival difference disappeared (22). Of note, as NAFLD-HCC patients often don't have cirrhosis, their liver function can be preserved compared to other etiologies, so both curative and palliative therapies may still be appropriate for some $(13,22,27,28)$. However, NAFLD-HCC patients are typically older, with a higher prevalence of T2DM, obesity, cardiovascular disease and cerebrovascular disease, which also need to be considered (13,27-29). Studies suggest that only a relatively small proportion of NAFLD-HCC patients are offered liver resection or orthotopic liver transplantation (OLT) $(17.8 \%$ and $4.4 \%$ respectively) and are more likely to be offered supportive care than those with other underlying etiologies $(22,27,28)$. Of note, although obesity and a raised BMI are associated with progression to cirrhosis in patients with NAFLD and generally poorer outcomes in those with the metabolic syndrome, body mass index (BMI) is positively associated with improved survival in HCC regardless of underlying etiology (28). This is very much in keeping with the marked negative impact that weight loss, constitutional symptoms and declining performance status are known to have on prognosis in patients with HCC (30).

\section{Biology of NAFLD-HCC}

The underlying pathophysiology of NAFLD-HCC is complex and the current understanding has been recently reviewed elsewhere $(31,32)$. Chronic hepatitis, necroinflammation and systemic metabolic dysfunction are all relevant, with proposed mechanisms including oxidative stress and DNA damage, dysfunctional DNA damage responses, dysfunctional autophagy, altered microbiome and innate and adaptive immune responses (31). In brief, fatty acid accumulation induces de novo lipogenesis and steatosis, as well as fatty acid oxidation associated with the production of reactive oxygen species (ROS) and DNA damage (33). DNA damage results in hepatocyte death, driving an inflammatory response with macrophages and neutrophils potentially releasing more ROS, causing further DNA damage $(31,34)$. Aberrant DNA damage repair responses may contribute to a permissive environment in which acquired genetic mutations promote HCC development $(35,36)$. Cellular proteins can also be damaged and autophagy is the process by which cytoplasmic proteins are tagged, transported to and degraded by lysosomes in response to metabolic stress-recycling in addition to removing them, supporting the energy requirement of the cell (37). In the context of NAFLD, excess triglycerides and free fatty acids have been shown to inhibit autophagy, again leading to increased cellular stress and DNA damage (38). Microbiome dysbiosis is another factor considered important in NAFLD progression, with some species (Firmicutes, Prevotellaceae and Enterobacteriaceae bacteria) more prevalent in patients with NASH (39) and roles in hepatocarcinogenesis suspected. Animal models support a contribution from the gut, with increased intestinal permeability and leakiness potentially promoting the translocation of lipopolysaccharide (LPS) to the liver, exacerbating inflammation and driving disease progression and NAFLD-HCC (40). Finally, underpinning the range in disease severity, patient genetic factors, with variations in liver regulatory genes such as PNPLA3, TM6SF2 and MBOAT7, are believed to play a key role not just in NAFLD progression, but also in determining an individual's risk of developing NAFLD-HCC (41-44).

\section{HCC surveillance in NAFLD}

Current international guidelines recommend screening for HCC in all patients with cirrhosis or advanced fibrosis with 6 monthly abdominal ultrasound (US) with or without alpha fetoprotein measurements (45-48). Surveillance in patients with NAFLD is often suboptimal, with up to $52 \%$ of NAFLD-HCC cases not diagnosed by surveillance, presenting with liver-related complications instead (22). The failure of surveillance in this population more so than others can be attributed to a number of factors. In patients with known NAFLD cirrhosis, abdominal US is not as sensitive as an early detection tool compared to other etiologies, as the presence of fatty liver disease and obesity hamper performance (49). Furthermore, in many patients with NAFLD cirrhosis and HCC, the presence of cirrhosis is only apparent at the time of HCC diagnosis and the opportunity for surveillance and early stage detection missed. Finally, the proportion of NAFLD-HCC which arises in the absence of cirrhosis is in part to blame, as surveillance in non-cirrhotic NAFLD is not recommended (45). This is because it is simply not cost effective and surveillance can 
cause harm (50). With the aim of reducing disease related mortality, cost effectiveness is influenced by the incidence of the disease in the target population, the availability of an acceptable and efficient diagnostic test at a reasonable cost, as well as the availability of effective treatments. Focused primarily on incidence, modelling studies have suggested that an HCC incidence of $1.5 \%$ per year or greater warrants surveillance in cirrhotic patients fit for resection (51). HCC incidence in NAFLD cirrhosis has been estimated to be in the region of $2.6 \%$ per year (19) and thus HCC surveillance justifiable. In the absence of cirrhosis with any etiology, the incidence is smaller, but patients may be able to withstand more radical therapies. Consequently an incidence in the region of $0.2 \%$ per year has been proposed as cost effective and on this basis, surveillance in non-cirrhotic patients with advanced or 'Metavir 3' fibrosis regardless of etiology has been advised, as has surveillance in non-cirrhotic HBV, with scores based on age, gender, platelet count and family history proposed to stratify risk (45). The challenge with NAFLD in the absence of significant fibrosis, is that the incidence is prohibitively small, estimated to be at most $0.01 \%$ per year in patients with simple steatosis (52), or $0.8 / 1,000$ person years in individuals with type 2 diabetes (53). The numbers of patients presenting with non-cirrhotic NAFLD-HCC reflects the enormous population exposed to this very small risk. Thus, there is a need for much cheaper and effective surveillance tests, as well as NAFLD patient specific data that will help to stratify the larger population and inform surveillance decisions. Risk calculations using age, gender and genotypes, such as PNPLA3 and TM6SF2 genotypes, are being researched but are not at present clinically useful $(50,54)$. Serum tests are cheaper than imaging and the GALAD serum score, comprised of a-fetoprotein, L3 $\alpha$-fetoprotein and des- $\gamma$-carboxyprothrombin has been shown to be superior to abdominal US for HCC detection in a retrospective cohort in the United States (55). Further prospective and surveillance studies are still awaited. Other novel candidate biomarkers include circulating tumor DNA (ctDNA), extra-cellular vesicles, serum Dickkopf 1 and serum osteopontin (31,56-58). Improving HCC surveillance in NAFLD would be the first step in improving outcomes, as it would increase the number of early stage and potentially curable tumours.

\section{HCC diagnosis in NAFLD}

HCCs commonly have a relatively arterialised blood supply relative to background liver, with arterial to venous shunting within the tumours. When these features are present on contrast enhanced imagining-detecting arterial hypervascularity and rapid venous washout, a radiological diagnosis of HCC is sensitive and specific in patients with cirrhosis (45). Due to the numbers of NAFLD patients developing HCC in the absence of cirrhosis, special consideration is needed with regards to diagnostic imaging. The radiological appearance of HCC in cirrhotic and noncirrhotic livers is generally similar, although the non-cirrhotic HCC are more often solitary without satellite lesions, larger and can have a central scar (59). Computer tomography (CT) imaging using properly administered contrast is essential for accurate assessment and can make the diagnosis of cancer with a high degree of confidence. Arterial enhancement and portal phase 'wash-out' is similar to cirrhotic livers, with features such as encapsulation, intralesional fat and foci of haemorrhage being more common (60). Despite these appearances, the probability of a 'typical lesion' being an HCC-rather than another type of cancer-is not as high as in the presence of cirrhosis. Thus, the nature of the malignancy cannot be assumed in the absence of cirrhosis. Any patient with a suspected malignancy in a non-cirrhotic liver, that is fit for therapy, should have a biopsy of the lesion to confirm the histological diagnosis. Distinguishing suspected cancers from benign lesions can also be challenging. $50 \%$ of non-cirrhotic HCC have a central scar-a feature often noted in the benign lesion focal nodal hyperplasia. Central scars are best characterised on MRI imaging using gadolinium. MRI is also better at detecting intracellular fat accumulation that may favour malignancy and is present in $10-17 \%$ of non-cirrhotic HCC and $36 \%$ of well differentiated tumours. Primovist MRI may help to increase the level of confidence that a lesion is benign, but generally the lack of central scar enhancement and the presence of satellite lesions on MRI, regardless of contrast agent, reportedly has a $98 \%$ specificity for malignancy (61). In summary, biopsy diagnosis is more common in patients with NAFLD-HCC. If there is any doubt that a lesion is benign, a biopsy should be considered. If a lesion in a noncirrhotic liver is thought to be an HCC, biopsy confirmation should be performed regardless of the imaging.

\section{NAFLD-HCC_implications for management}

\section{Staging of HCC in patients with NAFLD-HCC}

The management of HCC is complex and a multidisciplinary approach is vital. The main factors that 


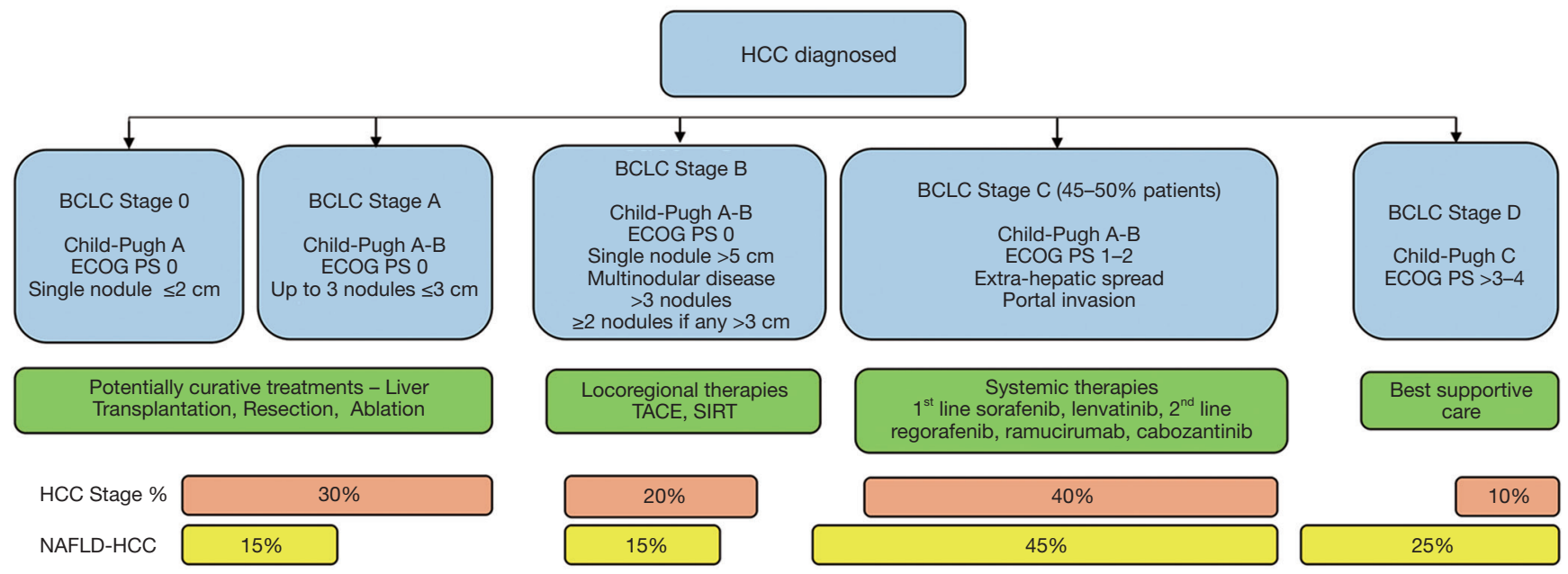

Figure 1 Hepatocellular carcinoma staging and treatment. The EASL guidelines recommend the BCLC algorithm for the management of patients with HCC (45). Patients are classified based on tumour stage, liver function and performance status into four categories (A-D) (blue boxes), with treatments ranging from resection, transplantation and ablation in fitter patients with early disease (BCLC-A), to supportive care in those with advanced HCC in association with poor liver function and/or performance status (BCLC-D) (green boxes). In the populations where HCV cirrhosis is highly prevalent (orange boxes), up to half the patients are suitable for curative or locoregional therapies, with $40 \%$ suitable for $1^{\text {st }}$ line medical therapy. In regions where NAFLD is the commonest cause of HCC, the majority of treatable patients are classed BCLC-C (13) and may have age or metabolic syndrome co-morbidities less suitable for medical therapies (yellow boxes). The recent updated EASL guideline supports ECOG-PS stage being attributed to cancer associated symptoms only, potentially enabling an allocation of a patient with physical restrictions impacting performance to BCLC-A or BCLC-B categories, if they have preserved liver function and an earlier stage cancer. In reality, there is little evidence supporting the use of locoregional therapies in these patients. Neither are there evidence based guidelines directing therapies in the absence of cirrhosis. Medical therapies should be considered, but neither elderly patients nor NAFLD patients have been well represented in the clinical trials of medical therapies. HCC, hepatocellular carcinoma; BCLC, Barcelona Clinic for Liver Cancer; EASL, European Association for the Study of the Liver; ECOG-PS, Eastern Cooperative Oncology Group Performance Status; TACE, transarterial chemoembolization; SIRT, selective internal radiotherapy treatment; NAFLD, non-alcoholic fatty liver disease.

determine management are stage of disease, tumor burden, liver function and performance status. The staging system widely used is the BCLC system, which comprises of 5 stages; very early disease (0), early stage (A), intermediate stage (B), advanced stage (C) and terminal stage (D). Tumor burden is categorized by the number and size of cancers, as well as presence of tumour macro vascular invasion and extra hepatic spread. Liver function is categorized using the Child-Turcotte-Pugh score, which is a three-tier scale from A to $\mathrm{C}$, with $\mathrm{C}$ indicating poor liver function. Performance status is categorized using the Eastern Cooperative Oncology Group Performance Status (ECOG-PS) (45). HCC staging and management is summarized in Figure 1.

Curative approaches for early stage disease with preserved liver function include liver resection and ablation, while liver transplantation can be used for early stage disease with poor liver function or portal hypertension. The commonest first line therapy is transarterial chemo-embolization, offered to those with intermediate stage disease and preserved liver function. For those with advanced cancers and with preserved liver function, systemic therapies with multikinase inhibitors sorafenib and lenvatinib are now available first line, while regorafenib, cabozantinib and ramucirumab are approved for selected individuals in the second line setting. For those with poor liver function and performance status, the majority are managed with best supportive care $(45,46)$, as liver transplantation is restricted to those fit enough with early stage cancers.

The relevance for the BCLC in guiding the management of NAFLD-HCC has been recently reviewed, highlighting both its strengths and weaknesses (31). Regardless of tumour stage, NAFLD-HCC patients more often don't have cirrhosis, but they do tend to be older, diagnosed at a more advanced age and with comorbidities such as obesity, 
T2DM, cardiovascular disease and cerebral vascular disease $(13,28)$. These are very relevant factors to consider when offering treatments, although their impact is not defined clearly within the BCLC algorithm, which was developed primarily as a tool to guide management for patients with viral hepatitis. The prognostic value of the BCLC across all etiologies is not in doubt, but the majority of NAFLDHCC patients fall into the heterogenous BCLC-C category, which directs patients to medical therapy, or the BCLC-D category directing to supportive care. Classifying the majority of NAFLD-HCC patients fit for treatment as 'BCLC-C' may be appropriate in prognostic terms, but the evidence base to guide treatment is limited. Elderly NAFLD-HCC patients have not been well represented in trials of medical therapy and if medical therapies are offered, efforts should be made to monitor 'real life practice' (62). In reality, some BCLC-C patients with preserved liver function and a small tumour burden may benefit more from selected loco-regional therapies, but these too lack an evidence base and further data is needed. In a large tertiary referral centre in Newcastle-upon-Tyne, where the majority of patients with HCC referred have either underlying NAFLD, or another etiology in combination with the metabolic syndrome, we have devised a modified management algorithm, which is described in Figure 2.

As NAFLD-HCC often presents in the absence of cirrhosis, liver function in these patients may be well preserved, even in advanced disease $(13,22)$. While some will have significant co-morbidities making them unsuitable for surgical or logoregional therapies, others may be fitter. Potentially therefore, the BCLC algorithm may well be too conservative. Increasingly there have been moves to 'downstage' patients with HCC, which is a term which typically refers to reducing the size of a tumour with transarterial therapy in order to consider liver transplantation. While transplantation is reserved for patients with cirrhosis, this downstaging principal can be applied in non-cirrhotic patients, who may subsequently have a lesser size or number of tumours, with residual disease suitable for treatment with ablation or resection. Thus, a patient treated initially with transarterial chemoembolization (TACE) may have ablation with curative intent to residual disease. Similarly, a non-cirrhotic patients with a large HCC may have selective internal radiotherapy treatment (SIRT) to reduce the size of their cancer in order to subsequently resect it (63). There is no randomised control trial evidence base for these practices and it is essential that management decisions are made within the forum of a multidisciplinary meeting (MDM), with the opinions of expert physicians, surgeons, radiologists and oncologists considered (Figure 2).

The major focus on management hereafter, is centred on those patients being considered for potentially curative therapies, with brief reference to evidence, or lack of it, for treating patients with more advanced NAFLD-HCC.

\section{Liver resection}

The criteria for resecting hepatocellular cancer in patients with cirrhosis is well defined and includes those patients with non-clinically significant portal hypertension (hepatic vein pressure gradient of $<10 \mathrm{mmHg}$ ), early stage disease (BCLC stage 0 to A), good performance status and preserved liver function. In the absence of cirrhosis, the criteria are less well defined. Since staging systems like the BCLC are associated with underlying cirrhosis, they are not relevant in these patients and tumour features are used for staging and prognosis. NAFLD-HCC patients more often than not, present with a more advanced stage of disease, with larger tumors which are technically more difficult to resect due to anatomical constraints or extra-hepatic disease making them un-resectable. This growing category of patients with non-cirrhotic NAFLD-HCC will also have metabolic syndrome co-morbidities—such as obesity, T2DM and cardiovascular disease-potentially increasing the risk of perioperative complications. These include bleeding, wound infection, delayed wound healing, venous thrombotic disease and pulmonary complications (64). In some experienced centres, up to $20 \%$ of NAFLD-HCC patients may undergo liver resection (22), although literature available on outcome from liver resection in patients with the metabolic syndrome is not, as yet comprehensive enough to inform evidence based guidelines.

A number of studies have reported comparable shortterm mortality in patients with either NAFLD or obesity following liver resection, as compared to those with other etiologies (29,65-71). In a multi-centre, retrospective study of HCC patients undergoing liver resection, 90 day mortality and morbidity of metabolic syndrome related HCC (MS-HCC) was comparable to that of HCV-HCC despite the significantly higher prevalence of obesity and T2DM (69). In fact, MS-HCC resection patients had better overall survival compared to HCV-HCC patients at $65.6 \%$ vs. $61.4 \%$ at 5 -year respectively $(\mathrm{P}=0.031)$. Recurrence in MS-HCC patients was also lower compared to HCV-HCC patients ( $44.6 \%$ vs. $65.2 \%$ respectively, 


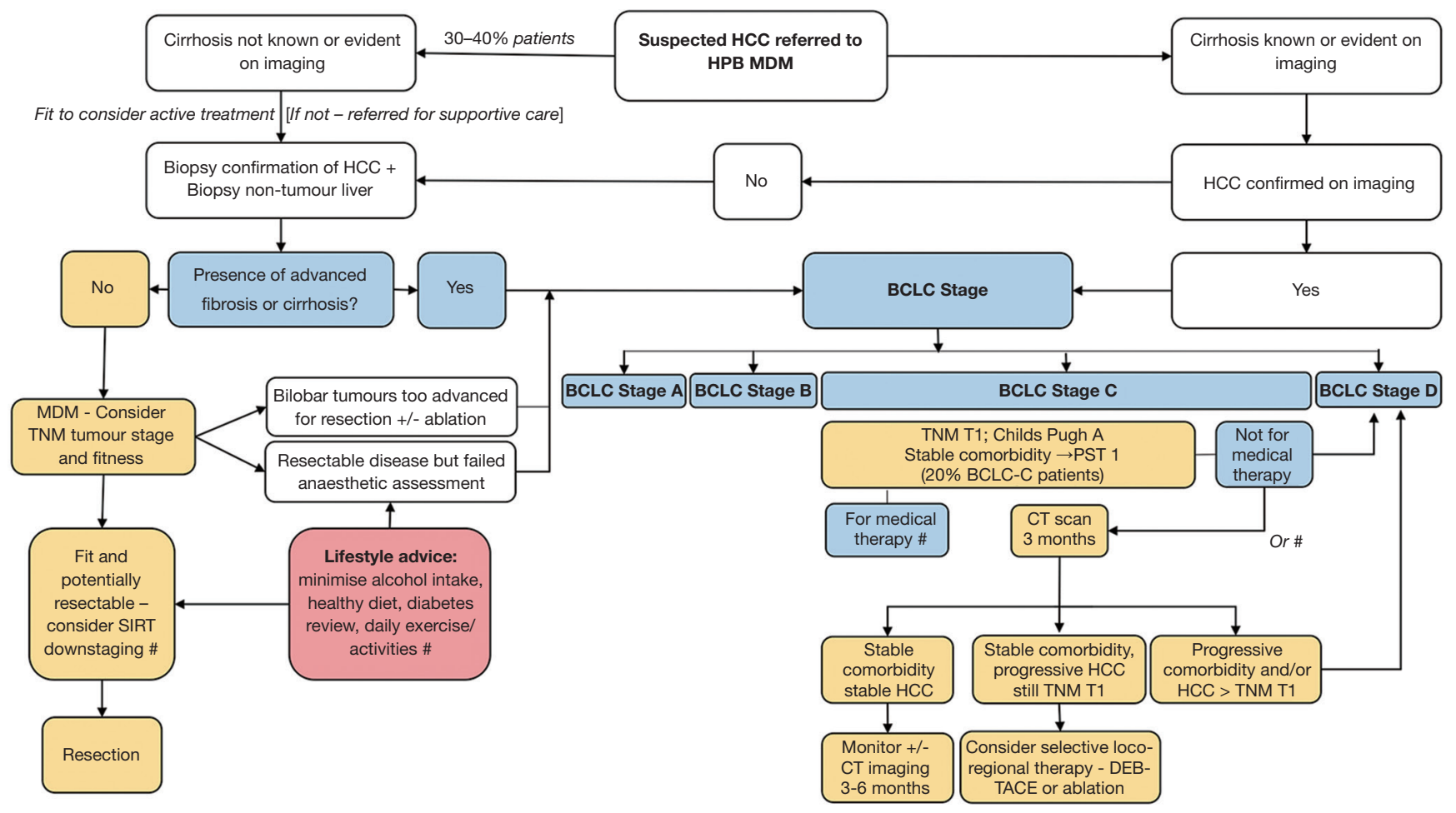

Figure 2 HCC MDT care in a centre with high prevalence of metabolic syndrome and NAFLD. In Newcastle upon Tyne, UK, NAFLD is the commonest cause of HCC and over two thirds of patients have the metabolic syndrome irrespective of their underlying liver disease etiology. NAFLD patients are more likely to present in the absence of cirrhosis and require biopsy confirmation of their HCC and liver disease stage. The BCLC algorithm guides treatment strategies in cirrhotic patients and in non-cirrhotic patients, liver function may be better preserved and more radical surgical approaches may be appropriate in those that are fit enough. In NAFLD patients classed as BCLC-C, medical therapies may not be ideal (e.g., restricted mobility, renal or cardiac impairment). Selective TACE may be better tolerated and preferable to supportive care in some instances. This figure summaries the modified BCLC pathway (yellow boxes) directed by the Newcastle upon Tyne Hepatopancreatobiliary multidisciplinary team (HPB MDT), with \# noting those areas where the evidence base is lacking and research or service improvement audits are necessary to inform future modifications to clinical practice. HPB, hepatobiliary; MDM, multidisciplinary meeting; HCC, hepatocellular carcinoma; BCLC, Barcelona Clinic for Liver Cancer; TNM, tumor node metastasis; ECOG-PS, Eastern Cooperative Oncology Group Performance Status; HCC, hepatocellular carcinoma; MDT, multidisciplinary team; SIRT, selective internal radiation therapy; DEB-TACE, drug-eluting bead transarterial chemoembolization; NAFLD, non-alcoholic fatty liver disease.

$\mathrm{P}=0.005)$. Although the HCV-HCC group possibly had more poorly differentiated tumors, and the study predated the HCV DAA era potentially delivering viral eradication pre or post resection, it was notable that having the metabolic syndrome per se did not appear to negatively impact prognosis. From multivariate analysis, MS-HCC was reported as a protective factor from early recurrence and $\mathrm{HCV}-\mathrm{HCC}$ a negative prognostic factor for overall survival and relapse free survival (69). An independent study comparing outcomes post resection of patients with and without steatosis similarly reported encouraging results, with no significant differences in postoperative complication and mortality (70). The study was again a retrospective one, in which the steatosis group did have a slightly smaller tumor size, with a lower vascular invasion rate, which may have contributed to the better 5-year survival (70). Similar retrospective studies also report comparable perioperative and long-term outcomes in NAFLD-HCC compared to other etiologies following liver resection (29,71). As alluded to previously, there have been studies reporting greater intraoperative blood losses in obese compared to non-obese individuals (67), as well as increased wound 
infections (66), but even some of these studies found no differences in survival. The presence of type 2 diabetes has also been reported to increase the risk of sepsis and even liver failure after liver resection (72-74), although again, increases in these perioperative complications does not necessarily have an impact on long term outcome (75).

Reports from centres in the Far East have also advocated resection as the primary curative treatment, Japanese patients with a high BMI $\left(>25 \mathrm{~kg} / \mathrm{m}^{2}\right)$ have a better long term prognosis compared to those with a lower BMI $\left(<25 \mathrm{~kg} / \mathrm{m}^{2}\right)$, with no increased risk of postoperative complications $(76,77)$. In series such as these it is unclear whether lower $\mathrm{BMI}$ is a surrogate marker for more advanced HCC, with HCC related cachexia and poor functional reserve. A large Chinese study found patients with a BMI $<18.5 \mathrm{~kg} / \mathrm{m}^{2}$ (underweight) or BMI $>25 \mathrm{~kg} / \mathrm{m}^{2}$ (overweight) had a worse prognosis to patients with a BMI of 18.5$25 \mathrm{~kg} / \mathrm{m}^{2}$ (normal weight) (78). These data are notable, but the number of patients with a BMI $>30 \mathrm{~kg} / \mathrm{m}^{2}$ was low in these studies, while it is relatively common in some western populations-where surgery on individuals with BMI $<18.5 \mathrm{~kg} / \mathrm{m}^{2}$ is rare. These studies may not be relevant to westernised populations.

In addition to these encouraging, if somewhat limited, results referred to in the studies above, there have been studies that have reported poorer short-term outcomes for patients with metabolic syndrome (79-84). One of these was of a large cohort of 3,973 patients undergoing liver resection, in which patients with metabolic syndrome had an increased incidence of blood transfusion, reintubation, myocardial infarction, surgical site infection as well as postoperative death (79). Smaller studies have also reported significantly higher 30-day mortality and morbidity in NAFLD-HCC patients compared to HCV and HBVHCC patients (82). Notably, several studies report increased morbidity and mortality in NAFLD patients with underlying NASH or cirrhosis compared to those with normal liver parenchyma (80-82). However, long-term outcomes in these studies were comparable. In NAFLD, careful surgical planning is needed in order to prevent post resection liver failure. Increased age, diabetes, steatosis and NASH are common in NAFLD-HCC patients and also increase the risk of post resection liver failure (85), possibly because these factors affect hepatic regeneration. The risk of post resection liver failure can be reduced with good preoperative planning utilizing qualitative and quantitative measures of liver function as well as the use of future remnant liver volume augmentation techniques such as portal vein embolization (85).

Thus, there is variation in the reported studies, which is not uncommon in retrospective case series and cohort studies which are affected by factors such as selection bias. It does appear though, that in experienced centres NAFLDHCC patients can do well post resection, particularly in the longer term. Thus, we should continue to explore and improve surgical options as well as tailor perioperative care to this complex group of patients. Laparoscopic approaches, which are better tolerated, with reduced intra-operative blood loss and reduced length of postoperative hospital stay, have been advocated by some centres (68).

While the focus here has largely been on factors most relevant to patients with the metabolic syndrome, generally speaking sarcopenia may be an alternative predictor of prognosis in HCC patients undergoing liver resection. A recent meta-analysis of 8 retrospective studies, including 1,161 patients of various etiologies undergoing either liver resection or radiofrequency ablation, reported that patients with sarcopenia had significantly increased rates of complications and tumour recurrence, as well as reduced 1- and 3-year survival (86). Whether or not it is possible to improve sarcopenia and positively impact outcomes is not yet known as very few studies have investigated the impact of interventions treating sarcopenia or improving nutritional status. One study investigating perioperative nutrition support in the form of enteral feeding reported a reduction in the length of hospital admission, but no difference in objective measures of morbidity or complications was demonstrated (87).

\section{Liver transplantation}

OLT is offered to patients who are deemed fit enough, with early stage cancers complicating cirrhosis, but in whom significant portal hypertension or impaired liver function is a barrier to safe resection. The tumour stage restriction most commonly applied is up to 3 nodules $\leq 3 \mathrm{~cm}$ or a single nodule $\leq 5 \mathrm{~cm}$ (88). OLT has the added benefit of curing the underlying liver disease. NAFLD cirrhosis is currently the second most common indication for OLT in the United States and is likely to become the leading indication in other western countries (89). Data from the European Transplant Registry shows an increase in the proportion of NAFLD transplants from $1.2 \%$ in 2002 to $8.4 \%$ in 2016 and shows that HCC is more common in patients transplanted for NAFLD compared to other eitiologies (90). From 1988 to 1996, $54 \%$ of patients receiving OLT in the United States 
were either overweight, obese, severely obese or morbidly obese (91). These figures are likely to be much higher now. As with liver resection, the generally older age of NAFLDHCC patients, along with presence of co-morbidities, does affect fitness for transplantation and as a result NAFLD patients are less likely to be receive OLT compared to other etiologies (92). In the Italian cohort of 145 NAFLD-HCC patients described earlier, only 1 received OLT (22).

For those that are transplanted, short term outcomes and postoperative complications are reportedly worse in NAFLD patients undergoing OLT, whether for end stage liver disease or for HCC. NAFLD patients tend to have longer operative time, increased blood loss and longer length of stay postoperatively (93-95). Historically, the presence of NASH has been shown to be an independent factor that influences early OLT mortality (96). In studies reporting higher early mortality in NAFLD patients, sepsis and cardiovascular disease are the most common causes of increased death (97-100). Interestingly NASH has been shown to be a risk factor for the development of cardiovascular complications following OLT independent to other comorbidities and metabolic factors $(99,101)$. A possible mechanism is that metabolic syndrome may stimulate erythrocyte hyperaggregability and hyperfibrinogenemia leading to a prothrombotic state which predisposes to thrombo-embolic and athero-thrombotic events (102). This emphasizes the importance of a tailored preoperative work up, including cardio-vascular testing. For OLT in the context of HCC, overweight and obese patients have a significantly increased incidence of perioperative life threatening complications, however 90 day mortality is reportedly unaffected (95).

Overall survival at 5-years following OLT for NAFLDcirrhosis is comparable to other etiologies. Longer term post OLT survival is slightly lower for NAFLD-HCC compared to NAFLD-cirrhosis, with 5-year survival being $66.7 \%$ versus $72.5 \%$ respectively (103). NAFLD-HCC patients who undergo OLT do have a similar diseasefree and overall survival compared to other etiologies of HCC $(98,104,105)$. A large retrospective cohort study using United Network for Organ Sharing data from 2002 to 2012 demonstrated that 5 -year survival post OLT for NAFLD, HCV and ARLD associated HCC was $65.5 \%$, $65.7 \%$ and $63.9 \%$ respectively (105). In a retrospective study of 159 patients undergoing OLT for HCC patients who were overweight or obese had a significantly increased rate of disease recurrence and reduced time to recurrence compared to patients of a normal weight (95). Diabetes also has a negative impact on outcomes with diabetes being associated with reduced $1-$ and 5-year overall survival as well as reduced graft survival (93). Furthermore, when diabetes co-exists with obesity, this further reduces 5 -year survival rates and this is particularly apparent in patients with HCC (93).

A recent analysis of 68,950 liver transplants recipients between 2002 and 2016 using data from the European Liver Transplant Registry database has also reported reassuring results (90). Overall there was comparable patient and graft survival post OLT between NAFLD and non-NAFLD patients, with and without HCC. Recurrent HCC, infection and extrahepatic solid organ malignancy were the most common causes of death in NAFLD-HCC patients whereas infection and cardiovascular complications were the most common in NAFLD non-HCC patients. In NAFLD non-HCC patients, older age, Model for EndStage Liver Disease (MELD) score $>23$ and BMI $\leq 18.5$ or $>40 \mathrm{~kg} / \mathrm{m}^{2}$ were independent factors associated with death. Interestingly this association was not seen in NAFLD-HCC patients. Unfortunately, data on metabolic risk factors such as the presence of T2DM, hypertension, hyperlipidemia and cardiovascular disease were missing from the dataset (90).

These studies suggest that although NAFLD patients may be at increased risk of short-term complications post OLT compared to other etiologies, patients with NAFLD-HCC have comparable longer-term outcomes to those of other etiologies underlying their HCC. With the aim of reducing short term complications, the focus in many centres is on prehabilitation, optimising fitness, weight and glycaemic control in the work up to OLT (Figure 2). Unfortunately, despite good outcomes recurrence of NAFLD following OLT is common and may affect up to $39 \%$ of grafts (106). Preventing this by managing metabolic risk factors post-transplant is another important management step.

Another important consideration is the effect of the growing prevalence of NAFLD on the quality of donor grafts. With NAFLD affecting around $25 \%$ of the world's population (16) and the demand for organs increasing, the number of steatotic allografts has increased (107). These steatotic organs are being used more frequently as extended criteria donors. Steatotic grafts are associated with poorer graft function and reduced graft survival (108). Other than liver biopsy, which is liable to sampling error and increasing cold ischaemia time while reporting is awaited, there are 
no validated tools to quantify steatosis. Better methods of detecting steatosis are needed in order to improve graft selection in a population with a high prevalence of NAFLD.

\section{Percutaneous ablation}

Percutaneous ablation is offered to patients with early stage disease but who are not suitable for surgery. Ablation is usually US guided and in patients with small single tumors has comparable efficacy to resection with far fewer complications (109). Ablative options include radiofrequency ablation, microwave ablation, irreversible electroporation and ethanol ablation. In theory NAFLDHCC patients may have worse outcomes with ablation because of the difficulties of US in overweight and obese patients. US beams can become attenuated by intrahepatic and subcutaneous fat potentially increasing the risk of complications and reducing efficacy.

Comparable outcomes for percutaneous ablation were reported in 2007 , in a prospective study comparing overweight (BMI $>25 \mathrm{~kg} / \mathrm{m}^{2}$ ) HCC patients to those of normal weight (BMI $\left.\leq 25 \mathrm{~kg} / \mathrm{m}^{2}\right)(110)$. The 219 overweight patients needed significantly more radiofrequency ablation sessions, as compared to 524 normal weight patients, but there were no differences in complication rates, recurrence rates, tumour progression and survival at 1,3 and 5 years (110). This study suggested that while percutaneous ablation may be more technically challenging in overweight patients, this did not affect outcome. The impact of advances in ablation technology, with techniques delivering more effective ablation and the wider use of CT rather than US guided ablation, have not formally been reported for obese, NAFLD patients per se, but these advances are ones likely to be of benefit for all.

Although much of the literature on the topic is rather dated, patients with diabetes undergoing percutaneous radiofrequency ablation have been reported to have a worse overall survival compared to patients without diabetes $(111,112)$. Diabetic patients taking metformin reportedly have a significantly better survival post ablation compared to diabetic patients not taking metformin, although this benefit is likely attributed to 'non-diabetic' benefits of metformin, rather than being directly attributable ablation or glycaemic control at the time of ablation $(111,112)$.

\section{TACE}

Patients with intermediate stage HCC (BCLC B) and good performance status can be offered TACE. TACE involves intra-arterial infusion of a cytotoxic agent followed by embolization of the tumour blood vessels. HCC unlike normal hepatic tissue gets its blood supply from branches of the hepatic artery, therefore TACE generally spares normal hepatic tissue and preserves liver function (113). As described previously, within a tertiary centre HCC MDT, selected patients with BCLC-C HCC may also be offered therapies such as selective TACE with drug-eluting beads, but further studies are needed to define the roles of locoregional therapies in these patients. In general, the effectiveness of TACE in NAFLD-HCC has not been well studied. A small but relatively recent retrospective study of 57 patients undergoing TACE looked at radiological outcomes in overweight patients (BMI $>25 \mathrm{~kg} / \mathrm{m}^{2}$ ) compared to normal weight patients (BMI $\leq 25 \mathrm{~kg} / \mathrm{m}^{2}$ ) (114), suggesting that an elevated BMI was associated with both a higher rate of residual disease post treatment and a shorter time to radiological progression (114). While there may be differences in the biology of HCC associated with liver diseases associated with obesity versus viral hepatitis related cirrhosis, data is insufficient to impact clinical decision making.

\section{Systemic therapies and the relevance of concurrent anti diabetic treatments}

Patients with advanced HCC (BCLC C), or those with earlier stage disease but with recurrence or progression following other therapies, with normal or only mildly impaired functional status (ECOG-PS 1-2), may be offered systemic therapies. Currently the tyrosine kinases inhibitors sorafenib and lenvatinib are the first line therapies approved by the Food and Drug Administration (FDA) (115-117). The FDA has approved regorafenib as a second line therapy (118). Cabozantinib another tyrosine kinase inhibitor has also shown efficacy along with the VEGF receptor 2 monoclonal antibody ramucirumab (119). For patients fit enough according to international guidelines, the recommendations for systemic therapy are the same, regardless of the underlying etiology of HCC. It should be noted, however, that patients with NAFLD-HCC have not been well represented in these studies. Furthermore, combined analyses of the Sharp and Asia-pacific studies reported that benefit for sorafenib was much greater for those with HCV related HCC rather than for other etiologies (120). Going forward, it would be helpful to have more NAFLD-HCC specific data, to inform clinical 
decisions.

The impact of the metabolic syndrome and the relevance for patients with NAFLD-HCC given these systemic therapies is not clear. Metformin is commonly used to control T2DM in NAFLD-HCC patients and while it has been reported to improve outcomes in patients treated with radiofrequency ablation and offer protection against HCC recurrence, metformin may be associated with poorer outcomes in patients taking sorafenib (111,121-123). Two studies have demonstrated that HCC patients taking long term metformin for diabetes have significantly worse progression free survival (PFS) and overall survival (OS) compared to patients not taking metformin $(121,122)$. In the larger of the studies, PFS and OS were 1.9 and 6.6 months respectively in patients taking metformin, compared to 3.7 and 10.8 months in patients not taking metformin $(\mathrm{P}<0.0001)$. Of note, diabetic patients not on metformin but taking insulin had better outcomes (8.4 months PFS and 16.6 months OS) (121).

Metformin is believed to have anti-tumor activity in chronic liver disease and HCC (112), with a poorer outcome for sorafenib in metformin treated patients perhaps surprising. Potential mechanisms have been considered and recently reviewed (124). Metformin and Sorafenib both potentially contribute to inhibition of mammalian target of rapamycin (mTOR). Metformin does so by the activation of AMP- activated protein kinase (AMPK), which positively regulates sirtuin-3 (SIRT-3) (125). SIRT-3 is a nicotinamide adenine dinucleotide $(\mathrm{NAD})^{+}$dependent deacetylase and is a key effector in the AMPK/HIF- $1 \alpha / \mathrm{mTOR}$ pathway, which regulates mitochondrial function, responses to oxidative stress and cell survival (125). Metformin rather than insulin treated patients reportedly have higher SIRT3 and mTOR levels (121) and it has been suggested that patients who develop HCC whilst taking metformin, may have acquired intrinsic resistance to the anti-tumour activity of metformin, and consequently to sorafenib, owing to their converging pathway of action $(121,124,126)$. This potential mechanism is not well understood (124) and as yet, there has been no published metformin based subgroup analysis from phase III studies showing sorafenib associated survival benefit (115-117). While further understanding may well be beneficial in the future, when considering the choice of medical therapy, there is no basis to discontinue the use of metformin in the pre-cancer or peri-cancer setting. Metformin may protect NAFLD patients from developing HCC and also has significant metabolic and cardiovascular benefits $(112,127-129)$.

\section{Novel therapies in clinical trials}

There are several novel therapies under investigation for the treatment of HCC, with much interest in immunotherapy approaches. Initial trials with immune checkpoint inhibitors demonstrated good safety and tolerance with some evidence of efficacy $(130,131)$. Although responses have been reported in all etiologies of HCC, which is potentially encouraging for patients with NAFLD-HCC, early indications from Phase III studies suggest failure as single agent therapies in 'all comer' trials and stratification biomarkers as well as combination approaches are likely to be needed. Combination approaches may include other standard therapies, such as locoregional therapies or medical therapies (sorafenib, lenvatinib), or possibly other immune therapies. Other immune approaches in pre-clinical trials include tumour vaccines, adoptive cell transfer with chimeric antigen $T$ cells, natural killer cells, tumor infiltrating lymphocytes and cytokine induced killer cells (132). Thus, the landscape is changing, with hope that medical therapies more effective or better tolerated for patients with NAFLD-HCC will be available within the next $5-10$ years.

\section{Conclusions}

NAFLD has become the leading cause of chronic liver disease worldwide and is becoming a leading cause of HCC. Patients with NAFLD-HCC tend to be older and have more co-morbidities than HCC of other etiologies. Due to the fact that HCC can arise in the absence of cirrhosis and that HCC surveillance programmes in NAFLD are suboptimal NAFLD-HCC tends to present at a late stage contributing to an overall poor prognosis. The late stage of presentation combined with patient age and co-morbidities reduce the use of curative therapies such as liver resection, OLT and ablation. Although NAFLD patients who undergo these therapies are at greater risk of perioperative complications, and therefore need careful preoperative assessment and optimisation, long term survival after treatment is comparable to other etiologies. The use of TACE and systemic therapies in NAFLD-HCC is not well studied but obesity may reduce the effectiveness of TACE and long-term metformin use may be associated with tumour resistance to sorafenib. More studies are needed to further evaluate this effect and to investigate if this association extends to other systemic therapies.

Currently clinical guidelines have a common algorithm for all etiologies of HCC, of which NAFLD has 
traditionally only made up a small part of the evidence base. As the prevalence of NAFLD-HCC continues to increase globally the need to update clinical guidelines for this specific group will become apparent. Prevention of NAFLD-HCC is a priority. This will involve public health measures to reduce metabolic risk factors, improve tools to screen for NAFLD and screen for HCC in those with established NAFLD including those without cirrhosis.

\section{Acknowledgments}

Funding: None.

\section{Footnote}

Reporting Checklist: The authors have completed the Narrative Review reporting checklist. Available at https://hbsn.amegroups.com/article/view/10.21037/ hbsn.2019.08.08/rc

Conflicts of Interest: All authors have completed the ICMJE uniform disclosure form (available at https://hbsn. amegroups.com/article/view/10.21037/hbsn.2019.08.08/coif). The authors have no conflicts of interest to declare.

Ethical Statement: The authors are accountable for all aspects of the work in ensuring that questions related to the accuracy or integrity of any part of the work are appropriately investigated and resolved.

Open Access Statement: This is an Open Access article distributed in accordance with the Creative Commons Attribution-NonCommercial-NoDerivs 4.0 International License (CC BY-NC-ND 4.0), which permits the noncommercial replication and distribution of the article with the strict proviso that no changes or edits are made and the original work is properly cited (including links to both the formal publication through the relevant DOI and the license). See: https://creativecommons.org/licenses/by-nc-nd/4.0/.

\section{References}

1. El-Serag HB, Rudolph KL. Hepatocellular carcinoma: epidemiology and molecular carcinogenesis.

Gastroenterology 2007;132:2557-76.

2. El-Serag HB. Hepatocellular carcinoma. N Engl J Med 2011;365:1118-27.

3. El-Serag HB, Davila JA, Petersen NJ, et al. The continuing increase in the incidence of hepatocellular carcinoma in the United States: an update. Ann Intern Med 2003;139:817-23.

4. Ryerson AB, Eheman CR, Altekruse SF, et al. Annual Report to the Nation on the Status of Cancer, 1975-2012, featuring the increasing incidence of liver cancer. Cancer 2016;122:1312-37.

5. Cancer Research UK. Liver cancer mortality statistics. Accessed 25 April 2019. Available online: https://www. cancerresearchuk.org/health-professional/cancerstatistics/statistics-by-cancer-type/liver-cancer/ mortality\#heading-Zero

6. $\mathrm{Xu}$ J. Trends in Liver Cancer Mortality Among Adults Aged 25 and Over in the United States, 2000-2016. NCHS Data Brief 2018;(314):1-8.

7. Jemal A, Bray F, Center MM, et al. Global cancer statistics. CA Cancer J Clin 2011;61:69-90.

8. Yang JD, Roberts LR. Hepatocellular carcinoma: A global view. Nat Rev Gastroenterol Hepatol 2010;7:448-58.

9. Petrick JL, Braunlin M, Laversanne M, et al. International trends in liver cancer incidence, overall and by histologic subtype, 1978-2007. Int J Cancer 2016;139:1534-45.

10. Benedict $M$, Zhang $X$. Non-alcoholic fatty liver disease: An expanded review. World J Hepatol 2017;9:715-32.

11. Kaur J. A comprehensive review on metabolic syndrome. Cardiol Res Pract 2014;2014:943162.

12. Younossi ZM, Koenig AB, Abdelatif D, et al. Global epidemiology of nonalcoholic fatty liver disease-Metaanalytic assessment of prevalence, incidence, and outcomes. Hepatology 2016;64:73-84.

13. Dyson J, Jaques B, Chattopadyhay D, et al. Hepatocellular cancer: the impact of obesity, type 2 diabetes and a multidisciplinary team. J Hepatol 2014;60:110-7.

14. Ertle J, Dechene A, Sowa JP, et al. Non-alcoholic fatty liver disease progresses to hepatocellular carcinoma in the absence of apparent cirrhosis. Int J Cancer 2011;128:2436-43.

15. Younossi Z, Stepanova M, Ong JP, et al. Nonalcoholic Steatohepatitis Is the Fastest Growing Cause of Hepatocellular Carcinoma in Liver Transplant Candidates. Clin Gastroenterol Hepatol 2019;17:748-55.e3.

16. Diehl AM, Day C. Cause, Pathogenesis, and Treatment of Nonalcoholic Steatohepatitis. N Engl J Med 2017;377:2063-72.

17. Starley BQ, Calcagno CJ, Harrison SA. Nonalcoholic fatty liver disease and hepatocellular carcinoma: a weighty connection. Hepatology 2010;51:1820-32.

18. Anderson EL, Howe LD, Jones HE, et al. The Prevalence 
of Non-Alcoholic Fatty Liver Disease in Children and Adolescents: A Systematic Review and Meta-Analysis. PLoS One 2015;10:e0140908.

19. Ascha MS, Hanouneh IA, Lopez R, et al. The incidence and risk factors of hepatocellular carcinoma in patients with nonalcoholic steatohepatitis. Hepatology 2010;51:1972-8.

20. Park JW, Chen M, Colombo M, et al. Global patterns of hepatocellular carcinoma management from diagnosis to death: the BRIDGE Study. Liver Int 2015;35:2155-66.

21. Younossi ZM, Otgonsuren M, Henry L, et al. Association of nonalcoholic fatty liver disease (NAFLD) with hepatocellular carcinoma (HCC) in the United States from 2004 to 2009. Hepatology 2015;62:1723-30.

22. Piscaglia F, Svegliati-Baroni G, Barchetti A, et al. Clinical patterns of hepatocellular carcinoma in nonalcoholic fatty liver disease: A multicenter prospective study. Hepatology 2016;63:827-38.

23. Mittal S, El-Serag HB, Sada YH, et al. Hepatocellular Carcinoma in the Absence of Cirrhosis in United States Veterans is Associated With Nonalcoholic Fatty Liver Disease. Clin Gastroenterol Hepatol 2016;14:124-31.e1.

24. Kawamura Y, Arase Y, Ikeda K, et al. Large-scale longterm follow-up study of Japanese patients with nonalcoholic Fatty liver disease for the onset of hepatocellular carcinoma. Am J Gastroenterol 2012;107:253-61.

25. Wong CR, Nguyen MH, Lim JK. Hepatocellular carcinoma in patients with non-alcoholic fatty liver disease. World J Gastroenterol 2016;22:8294-303.

26. Mittal S, Sada YH, El-Serag HB, et al. Temporal trends of nonalcoholic fatty liver disease-related hepatocellular carcinoma in the veteran affairs population. Clin Gastroenterol Hepatol 2015;13:594-601.e1.

27. Than NN, Ghazanfar A, Hodson J, et al. Comparing clinical presentations, treatments and outcomes of hepatocellular carcinoma due to hepatitis $\mathrm{C}$ and nonalcoholic fatty liver disease. QJM 2017;110:73-81.

28. Weinmann A, Alt Y, Koch S, et al. Treatment and survival of non-alcoholic steatohepatitis associated hepatocellular carcinoma. BMC Cancer 2015;15:210.

29. Pais R, Fartoux L, Goumard C, et al. Temporal trends, clinical patterns and outcomes of NAFLD-related HCC in patients undergoing liver resection over a 20-year period. Aliment Pharmacol Ther 2017;46:856-63.

30. Llovet JM, Bustamante J, Castells A, et al. Natural history of untreated nonsurgical hepatocellular carcinoma: rationale for the design and evaluation of therapeutic trials. Hepatology 1999;29:62-7.
31. Anstee QM, Reeves HL, Kotsiliti E, et al. From NASH to HCC: current concepts and future challenges. Nat Rev Gastroenterol Hepatol 2019;16:411-28.

32. Kutlu O, Kaleli HN, Ozer E. Molecular Pathogenesis of Nonalcoholic Steatohepatitis- (NASH-) Related Hepatocellular Carcinoma. Can J Gastroenterol Hepatol 2018;2018:8543763.

33. Perla FM, Prelati M, Lavorato M, et al. The Role of Lipid and Lipoprotein Metabolism in Non-Alcoholic Fatty Liver Disease. Children (Basel) 2017;4:46.

34. Yuan D, Huang S, Berger E, et al. Kupffer Cell-Derived Tnf Triggers Cholangiocellular Tumorigenesis through JNK due to Chronic Mitochondrial Dysfunction and ROS. Cancer Cell 2017;31:771-89.e6.

35. Cornell L, Munck JM, Alsinet C, et al. DNA-PK-A candidate driver of hepatocarcinogenesis and tissue biomarker that predicts response to treatment and survival. Clin Cancer Res 2015;21:925-33.

36. Daugherity EK, Balmus G, Al Saei A, et al. The DNA damage checkpoint protein ATM promotes hepatocellular apoptosis and fibrosis in a mouse model of non-alcoholic fatty liver disease. Cell Cycle 2012;11:1918-28.

37. Dikic I, Elazar Z. Mechanism and medical implications of mammalian autophagy. Nat Rev Mol Cell Biol 2018;19:349-64.

38. Kim J, Kundu M, Viollet B, et al. AMPK and mTOR regulate autophagy through direct phosphorylation of Ulk1. Nat Cell Biol 2011;13:132-41.

39. Zhu L, Baker SS, Gill C, et al. Characterization of gut microbiomes in nonalcoholic steatohepatitis (NASH) patients: a connection between endogenous alcohol and NASH. Hepatology 2013;57:601-9.

40. Gäbele E, Dostert K, Hofmann C, et al. DSS induced colitis increases portal LPS levels and enhances hepatic inflammation and fibrogenesis in experimental NASH. J Hepatol 2011;55:1391-9.

41. Donati B, Dongiovanni P, Romeo S, et al. MBOAT7 rs641738 variant and hepatocellular carcinoma in noncirrhotic individuals. Sci Rep 2017;7:4492.

42. Liu YL, Patman GL, Leathart JB, et al. Carriage of the PNPLA3 rs738409 $\mathrm{C}>\mathrm{G}$ polymorphism confers an increased risk of non-alcoholic fatty liver disease associated hepatocellular carcinoma. J Hepatol 2014;61:75-81.

43. Liu YL, Reeves HL, Burt AD, et al. TM6SF2 rs58542926 influences hepatic fibrosis progression in patients with non-alcoholic fatty liver disease. Nat Commun 2014;5:4309.

44. Singal AG, Manjunath H, Yopp AC, et al. The effect 
of PNPLA3 on fibrosis progression and development of hepatocellular carcinoma: a meta-analysis. Am J Gastroenterol 2014;109:325-34.

45. EASL Clinical Practice Guidelines: Management of hepatocellular carcinoma. J Hepatol 2018;69:182-236.

46. Heimbach JK, Kulik LM, Finn RS, et al. AASLD guidelines for the treatment of hepatocellular carcinoma. Hepatology 2018;67:358-80.

47. Omata M, Cheng AL, Kokudo N, et al. Asia-Pacific clinical practice guidelines on the management of hepatocellular carcinoma: a 2017 update. Hepatol Int 2017;11:317-70.

48. Vogel A, Cervantes A, Chau I, et al. Hepatocellular carcinoma: ESMO Clinical Practice Guidelines for diagnosis, treatment and follow-up. Ann Oncol 2018;29:iv238-55.

49. Del Poggio P, Olmi S, Ciccarese F, et al. Factors that affect efficacy of ultrasound surveillance for early stage hepatocellular carcinoma in patients with cirrhosis. Clin Gastroenterol Hepatol 2014;12:1927-33.e2.

50. Geh D, Rana FA, Reeves HL. Weighing the benefits of hepatocellular carcinoma surveillance against potential harms. J Hepatocell Carcinoma 2019;6:23-30.

51. Sarasin FP, Giostra E, Hadengue A. Cost-effectiveness of screening for detection of small hepatocellular carcinoma in western patients with Child-Pugh class A cirrhosis. Am J Med 1996;101:422-34.

52. Duan XY, Zhang L, Fan JG, et al. NAFLD leads to liver cancer: do we have sufficient evidence? Cancer Lett 2014;345:230-4.

53. Morling JR, Fallowfield JA, Guha IN, et al. Clinically significant chronic liver disease in people with Type 2 diabetes: the Edinburgh Type 2 Diabetes Study. QJM 2016;109:249-56.

54. Hassan MM, Kaseb A, Etzel CJ, et al. Genetic variation in the PNPLA3 gene and hepatocellular carcinoma in USA: risk and prognosis prediction. Mol Carcinog 2013;52 Suppl 1:E139-47.

55. Yang JD, Addissie BD, Mara KC, et al. GALAD Score for Hepatocellular Carcinoma Detection in Comparison with Liver Ultrasound and Proposal of GALADUS Score. Cancer Epidemiol Biomarkers Prev 2019;28:531-8.

56. Arbelaiz A, Azkargorta M, Krawczyk M, et al. Serum extracellular vesicles contain protein biomarkers for primary sclerosing cholangitis and cholangiocarcinoma. Hepatology 2017;66:1125-43.

57. Hwang A, Shi C, Zhu E, et al. Supervised learning reveals circulating biomarker levels diagnostic of hepatocellular carcinoma in a clinically relevant model of nonalcoholic steatohepatitis; An OAD to NASH. PLoS One 2018;13:e0198937.

58. Xu RH, Wei W, Krawczyk M, et al. Circulating tumour DNA methylation markers for diagnosis and prognosis of hepatocellular carcinoma. Nat Mater 2017;16:1155-61.

59. Winston CB, Schwartz LH, Fong Y, et al. Hepatocellular carcinoma: MR imaging findings in cirrhotic livers and noncirrhotic livers. Radiology 1999;210:75-9.

60. Di Martino M, Saba L, Bosco S, et al. Hepatocellular carcinoma (HCC) in non-cirrhotic liver: clinical, radiological and pathological findings. Eur Radiol 2014;24:1446-54.

61. Fischer MA, Raptis DA, Donati OF, et al. MR imaging features for improved diagnosis of hepatocellular carcinoma in the non-cirrhotic liver: Multi-center evaluation. Eur J Radiol 2015;84:1879-87.

62. King J, Palmer DH, Johnson P, et al. Sorafenib for the Treatment of Advanced Hepatocellular Cancer - a UK Audit. Clin Oncol (R Coll Radiol) 2017;29:256-62.

63. Moir JA, Burns J, Barnes J, et al. Selective internal radiation therapy for liver malignancies. Br J Surg 2015;102:1533-40.

64. Choban PS, Flancbaum L. The impact of obesity on surgical outcomes: a review. J Am Coll Surg 1997;185:593-603.

65. Anstee QM, Targher G, Day CP. Progression of NAFLD to diabetes mellitus, cardiovascular disease or cirrhosis. Nat Rev Gastroenterol Hepatol 2013;10:330-44.

66. Guo Z, Zhang J, Jiang JH, et al. Obesity Does Not Influence Outcomes in Hepatocellular Carcinoma Patients following Curative Hepatectomy. PLoS One 2015;10:e0125649.

67. Tanaka S, Iimuro Y, Hirano T, et al. Safety of hepatic resection for hepatocellular carcinoma in obese patients with cirrhosis. Surg Today 2013;43:1290-7.

68. Toriguchi K, Hatano E, Sakurai T, et al. Laparoscopic liver resection in obese patients. World J Surg 2015;39:1210-5.

69. Vigano L, Conci S, Cescon M, et al. Liver resection for hepatocellular carcinoma in patients with metabolic syndrome: A multicenter matched analysis with HCVrelated HCC. J Hepatol 2015;63:93-101.

70. Wu TH, Yu MC, Chan KM, et al. Prognostic effect of steatosis on hepatocellular carcinoma patients after liver resection. Eur J Surg Oncol 2011;37:618-22.

71. Yang T, Hu LY, Li ZL, et al. Liver Resection for Hepatocellular Carcinoma in Non-alcoholic Fatty Liver Disease: a Multicenter Propensity Matching Analysis with 
HBV-HCC. J Gastrointest Surg 2020;24:320-9.

72. Huo TI, Lui WY, Huang YH, et al. Diabetes mellitus is a risk factor for hepatic decompensation in patients with hepatocellular carcinoma undergoing resection: a longitudinal study. Am J Gastroenterol 2003;98:2293-8.

73. Matsumata T, Taketomi A, Kawahara N, et al. Morbidity and mortality after hepatic resection in the modern era. Hepatogastroenterology 1995;42:456-60.

74. Shirabe K, Shimada M, Gion T, et al. Postoperative liver failure after major hepatic resection for hepatocellular carcinoma in the modern era with special reference to remnant liver volume. J Am Coll Surg 1999;188:304-9.

75. Poon RT, Fan ST, Wong J. Does diabetes mellitus influence the perioperative outcome or long term prognosis after resection of hepatocellular carcinoma? Am J Gastroenterol 2002;97:1480-8.

76. Itoh S, Ikeda $\mathrm{Y}$, Kawanaka $\mathrm{H}$, et al. The effect of overweight status on the short-term and 20-y outcomes after hepatic resection in patients with hepatocellular carcinoma. J Surg Res 2012;178:640-5.

77. Okamura Y, Maeda A, Matsunaga K, et al. Negative impact of low body mass index on surgical outcomes after hepatectomy for hepatocellular carcinoma. J Hepatobiliary Pancreat Sci 2012;19:449-57.

78. Yu JJ, Shen F, Chen TH, et al. Multicentre study of the prognostic impact of preoperative bodyweight on longterm prognosis of hepatocellular carcinoma. Br J Surg 2019; 106:276-85.

79. Bhayani NH, Hyder O, Frederick W, et al. Effect of metabolic syndrome on perioperative outcomes after liver surgery: A National Surgical Quality Improvement Program (NSQIP) analysis. Surgery 2012;152:218-26.

80. Cauchy F, Zalinski S, Dokmak S, et al. Surgical treatment of hepatocellular carcinoma associated with the metabolic syndrome. Br J Surg 2013;100:113-21.

81. Reddy SK, Marsh JW, Varley PR, et al. Underlying steatohepatitis, but not simple hepatic steatosis, increases morbidity after liver resection: a case-control study. Hepatology 2012;56:2221-30.

82. Wakai T, Shirai Y, Sakata J, et al. Surgical outcomes for hepatocellular carcinoma in nonalcoholic fatty liver disease. J Gastrointest Surg 2011;15:1450-8.

83. Yoshida N, Takayama T, Midorikawa Y, et al. Surgical outcomes in patients with hepatocellular carcinoma associated with metabolic syndrome. World J Surg 2015;39:471-7.

84. Zarzavadjian Le Bian A, Costi R, Constantinides V, et al. Metabolic disorders, non-alcoholic fatty liver disease and major liver resection: an underestimated perioperative risk. J Gastrointest Surg 2012;16:2247-55.

85. Ray S, Mehta NN, Golhar A, et al. Post hepatectomy liver failure - A comprehensive review of current concepts and controversies. Ann Med Surg (Lond) 2018;34:4-10.

86. Zhang G, Meng S, Li R, et al. Clinical significance of sarcopenia in the treatment of patients with primary hepatic malignancies, a systematic review and metaanalysis. Oncotarget 2017;8:102474-85.

87. Yao H, Bian X, Mao L, et al. Preoperative Enteral Nutritional Support in Patients Undergoing Hepatectomy for Hepatocellular Carcinoma: A Strengthening the Reporting of Observational Studies in Epidemiology Article. Medicine (Baltimore) 2015;94:e2006.

88. Mazzaferro V, Regalia E, Doci R, et al. Liver transplantation for the treatment of small hepatocellular carcinomas in patients with cirrhosis. $\mathrm{N}$ Engl J Med 1996;334:693-9.

89. Wong RJ, Aguilar M, Cheung R, et al. Nonalcoholic steatohepatitis is the second leading etiology of liver disease among adults awaiting liver transplantation in the United States. Gastroenterology 2015;148:547-55.

90. Haldar D, Kern B, Hodson J, et al. Outcomes of liver transplantation for non-alcoholic steatohepatitis: A European Liver Transplant Registry study. J Hepatol 2019;71:313-22.

91. Nair S, Verma S, Thuluvath PJ. Obesity and its effect on survival in patients undergoing orthotopic liver transplantation in the United States. Hepatology 2002;35:105-9.

92. O'Leary JG, Landaverde C, Jennings L, et al. Patients with $\mathrm{NASH}$ and cryptogenic cirrhosis are less likely than those with hepatitis $\mathrm{C}$ to receive liver transplants. Clin Gastroenterol Hepatol 2011;9:700-4 e1.

93. Adams LA, Arauz O, Angus PW, et al. Additive impact of pre-liver transplant metabolic factors on survival post-liver transplant. J Gastroenterol Hepatol 2016;31:1016-24.

94. Agopian VG, Kaldas FM, Hong JC, et al. Liver transplantation for nonalcoholic steatohepatitis: the new epidemic. Ann Surg 2012;256:624-33.

95. Mathur A, Franco ES, Leone JP, et al. Obesity portends increased morbidity and earlier recurrence following liver transplantation for hepatocellular carcinoma. HPB (Oxford) 2013;15:504-10.

96. Barritt ASt, Dellon ES, Kozlowski T, et al. The influence of nonalcoholic fatty liver disease and its associated comorbidities on liver transplant outcomes. J Clin Gastroenterol 2011;45:372-8. 
97. Kennedy C, Redden D, Gray S, et al. Equivalent survival following liver transplantation in patients with nonalcoholic steatohepatitis compared with patients with other liver diseases. HPB (Oxford) 2012;14:625-34.

98. Malik SM, deVera ME, Fontes P, et al. Outcome after liver transplantation for NASH cirrhosis. Am J Transplant 2009;9:782-93.

99. Vanwagner LB, Bhave M, Te HS, et al. Patients transplanted for nonalcoholic steatohepatitis are at increased risk for postoperative cardiovascular events. Hepatology 2012;56:1741-50.

100. Wang X, Li J, Riaz DR, et al. Outcomes of liver transplantation for nonalcoholic steatohepatitis: a systematic review and meta-analysis. Clin Gastroenterol Hepatol 2014;12:394-402 e1.

101. Wong VW, Wong GL, Yip GW, et al. Coronary artery disease and cardiovascular outcomes in patients with nonalcoholic fatty liver disease. Gut 2011;60:1721-7.

102. Vaya A, Hernandez-Mijares A, Bonet E, et al. Association between hemorheological alterations and metabolic syndrome. Clin Hemorheol Microcirc 2011;49:493-503.

103.Afzali A, Berry K, Ioannou GN. Excellent posttransplant survival for patients with nonalcoholic steatohepatitis in the United States. Liver Transpl 2012;18:29-37.

104. Reddy SK, Steel JL, Chen HW, et al. Outcomes of curative treatment for hepatocellular cancer in nonalcoholic steatohepatitis versus hepatitis $\mathrm{C}$ and alcoholic liver disease. Hepatology 2012;55:1809-19.

105. Wong RJ, Chou C, Bonham CA, et al. Improved survival outcomes in patients with non-alcoholic steatohepatitis and alcoholic liver disease following liver transplantation: an analysis of 2002-2012 United Network for Organ Sharing data. Clin Transplant 2014;28:713-21.

106. Dureja P, Mellinger J, Agni R, et al. NAFLD recurrence in liver transplant recipients. Transplantation 2011;91:684-9.

107.Zezos P, Renner EL. Liver transplantation and nonalcoholic fatty liver disease. World J Gastroenterol 2014;20:15532-8.

108. Chu MJ, Dare AJ, Phillips AR, et al. Donor Hepatic Steatosis and Outcome After Liver Transplantation: a Systematic Review. J Gastrointest Surg 2015;19:1713-24.

109. Cucchetti A, Piscaglia F, Cescon M, et al. Costeffectiveness of hepatic resection versus percutaneous radiofrequency ablation for early hepatocellular carcinoma. J Hepatol 2013;59:300-7.

110. Ohki T, Tateishi R, Shiina S, et al. Obesity did not diminish the efficacy of percutaneous ablation for hepatocellular carcinoma. Liver Int 2007;27:360-7.
111. Chen TM, Lin CC, Huang PT, et al. Metformin associated with lower mortality in diabetic patients with early stage hepatocellular carcinoma after radiofrequency ablation. J Gastroenterol Hepatol 2011;26:858-65.

112. Donadon V, Balbi M, Mas MD, et al. Metformin and reduced risk of hepatocellular carcinoma in diabetic patients with chronic liver disease. Liver Int 2010;30:750-8.

113. Villanueva A. Hepatocellular Carcinoma. N Engl J Med 2019;380:1450-62.

114. Wu SE, Charles HW, Park JS, et al. Obesity conveys poor outcome in patients with hepatocellular carcinoma treated by transarterial chemoembolization. Diagn Interv Imaging 2017;98:37-42.

115.Cheng AL, Kang YK, Chen Z, et al. Efficacy and safety of sorafenib in patients in the Asia-Pacific region with advanced hepatocellular carcinoma: a phase III randomised, double-blind, placebo-controlled trial. Lancet Oncol 2009;10:25-34.

116. Kudo M, Finn RS, Qin S, et al. Lenvatinib versus sorafenib in first-line treatment of patients with unresectable hepatocellular carcinoma: a randomised phase 3 noninferiority trial. Lancet 2018;391:1163-73.

117.Llovet JM, Ricci S, Mazzaferro V, et al. Sorafenib in advanced hepatocellular carcinoma. N Engl J Med 2008;359:378-90.

118. Bruix J, Qin S, Merle P, et al. Regorafenib for patients with hepatocellular carcinoma who progressed on sorafenib treatment (RESORCE): a randomised, double-blind, placebo-controlled, phase 3 trial. Lancet 2017;389:56-66.

119.Zhu AX, Kang YK, Yen CJ, et al. Ramucirumab after sorafenib in patients with advanced hepatocellular carcinoma and increased alpha-fetoprotein concentrations (REACH-2): a randomised, double-blind, placebocontrolled, phase 3 trial. Lancet Oncol 2019;20:282-96.

120. Bruix J, Cheng AL, Meinhardt G, et al. Prognostic factors and predictors of sorafenib benefit in patients with hepatocellular carcinoma: Analysis of two phase III studies. J Hepatol 2017;67:999-1008.

121. Casadei Gardini A, Faloppi L, De Matteis S, et al. Metformin and insulin impact on clinical outcome in patients with advanced hepatocellular carcinoma receiving sorafenib: Validation study and biological rationale. Eur J Cancer 2017;86:106-14.

122. Casadei Gardini A, Marisi G, Scarpi E, et al. Effects of metformin on clinical outcome in diabetic patients with advanced HCC receiving sorafenib. Expert Opin Pharmacother 2015;16:2719-25. 
123.Zhou YY, Zhu GQ, Liu T, et al. Systematic Review with Network Meta-Analysis: Antidiabetic Medication and Risk of Hepatocellular Carcinoma. Sci Rep 2016;6:33743.

124. De Matteis S, Scarpi E, Granato AM, et al. Role of SIRT-3, p-mTOR and HIF-1alpha in Hepatocellular Carcinoma Patients Affected by Metabolic Dysfunctions and in Chronic Treatment with Metformin. Int J Mol Sci 2019;20:1503.

125. De Matteis S, Granato AM, Napolitano R, et al. Interplay Between SIRT-3, Metabolism and Its Tumor Suppressor Role in Hepatocellular Carcinoma. Dig Dis Sci 2017;62:1872-80.

126. Orsi G, Casadei-Gardini A. Sorafenib and metformin: to be, or not to be, that is the question. Hepatobiliary Surg Nutr 2019;8:411-3.

127. Francque SM, van der Graaff D, Kwanten WJ. Nonalcoholic fatty liver disease and cardiovascular risk: Pathophysiological mechanisms and implications. J

Cite this article as: Geh D, Manas DM, Reeves HL. Hepatocellular carcinoma in non-alcoholic fatty liver disease-a review of an emerging challenge facing clinicians. HepatoBiliary Surg Nutr 2021;10(1):59-75. doi: 10.21037/hbsn.2019.08.08
Hepatol 2016;65:425-43.

128. Rena G, Lang CC. Repurposing Metformin for Cardiovascular Disease. Circulation 2018;137:422-4.

129. Li Y, Liu L, Wang B, et al. Metformin in non-alcoholic fatty liver disease: A systematic review and meta-analysis. Biomed Rep 2013;1:57-64.

130.El-Khoueiry AB, Sangro B, Yau T, et al. Nivolumab in patients with advanced hepatocellular carcinoma (CheckMate 040): an open-label, non-comparative, phase 1/2 dose escalation and expansion trial. Lancet 2017;389:2492-502.

131. Sangro B, Gomez-Martin C, de la Mata M, et al. A clinical trial of CTLA-4 blockade with tremelimumab in patients with hepatocellular carcinoma and chronic hepatitis C. J Hepatol 2013;59:81-8.

132. Xie Y, Xiang Y, Sheng J, et al. Immunotherapy for Hepatocellular Carcinoma: Current Advances and Future Expectations. J Immunol Res 2018;2018:8740976. 